\title{
BIONOMIA E CHAVE PARA IMATUROS E ADULTOS DE Simulium (DIPTERA: SIMULIIDAE) NAAMAZÔNIA CENTRAL, BRASIL
}

\author{
Neusa HAMADA ${ }^{1}$, Peter H. ADLER ${ }^{2}$
}

RESUMO - O presente estudo teve como objetivos apresentar informações bionômicas e de distribuição sobre larvas e pupas de simulídeos na Amazônia Central, delimitada aqui como Manaus (AM) e municípios nos arredores (Presidente Figueiredo, Itacoatiara, Careiro da Várzea, Manacapuru, Novo Airão), assim como apresentar chaves para todos os estágios das espécies presentes na área. Amostrou-se 79 locais, que foram caracterizados com os seguintes parâmetros físico-químicos: largura, profundidade, correnteza, vazão, $\mathrm{pH}$, temperatura, vegetação predominante e presença de represamento d'água. Onze espécies foram coletadas nos igarapés dessa área (Simulium cauchense Floch \& Abonnenc, Simulium daltanhani Hamada \& Adler, Simulium goeldii Cerqueira \& Nunes de Mello, Simulium iracouboense Floch \& Abonnenc, Simulium maroniense Floch \& Abonnenc, Simulium perflavum Roubaud, Simulium quadrifidum Lutz, Simulium rorotaense Floch \& Abonnenc, Simulium trombetense Hamada, Py-Daniel \& Adler, Simulium "6-B1" e Simulium "A"). Simulium argentiscutum Shelley \& Luna Dias foi coletada apenas como fềmeas adultas, picando seres humanos no município de Itacoatiara. Chaves para larvas, pupas e adultos fêmeas e machos, com ilustrações de caracteres relevantes, das 12 espécies presentes na Amazônia Central, são fornecidas. Os imaturos de Simuliidae se distribuíram de maneira distinta, formando grupos de espécies diferenciados pelas caracteristicas medidas no criadouro.

Palavras-chave: Simulium, Simuliidae, Amazônia Central, taxonomia, bionomia

Bionomics and Keys to Immatures and Adults of Simulium (Diptera: Simuliidae) from Central Amazonia, Brazil.

ABSTRACT - The present study provides bionomic and distribution data on larvae and pupae of black flies in Central Amazonia, Brazil, defined here as Manaus (Amazonas) and nearby counties (Presidente Figueiredo, Itacoatiara, Careiro da Várzea, Manacapuru, Novo Airão). Keys are provided for larvae, pupae, females and males of black flies from this area. Seventy-nine localities were sampled, and they were characterized by the following physico-chemical parameters: water width, depth, velocity, discharge, $\mathrm{pH}$, temperature, predominant vegetation and presence of a dam. Eleven species were found in streams in this area (Simulium cauchense Floch \& Abonnenc, Simulium daltanhani Hamada \& Adler, Simulium goeldii Cerqueira \& Nunes de Mello, Simulium iracouboense Floch \& Abonnenc, Simulium maroniense Floch \& Abonnenc, Simulium perflavum Roubaud, Simulium quadrifidum Lutz, Simulium rorotaense Floch \& Abonnenc, Simulium trombetense Hamada, Py-Daniel \& Adler, Simulium "6-B1" and Simulium "A"). Simulium argentiscutum Shelley \& Luna Dias was collected only as females biting humans in Itacoatiara County. Keys for larvae, pupae, females and males, with illustrations of relevant characters are provided for all 12 species present in Central Amazonia. The immatures of Simuliidae were distributed in ways that were distinct to each species, forming groups of species differentiated by the measured habitat characteristics.

Key-words: Simulium, Simuliidae, Central Amazonia, taxonomy, bionomics

\section{INTRODUÇÃO}

\section{Os trabalhos realizados com}

simulídeos na Amazônia Central, definida aqui como Manaus (AM) e outros municípios nos seus arredores,

1. Instituto Nacional de Pesquisas da Amazônia, Coordenação de Pesquisas em Entomologia, Caixa Postal 478,69011-970 Manaus,AM, Brasil, E-mail: nhamada@inpa,gov,br

2. Department of Entomology, Clemson University Clemson, SC 29634, USA, E-mail: padler@clemson.edu 
têm se restringido, em geral, a aspectos ecológicos e biológicos de espécies individuais (Dellome Filho, 1978, 1983; Gorayeb \& Pinger, 1978; Gorayeb \& Mok, 1982; Lacey \& Lacey, 1983; Hamada, 1989; 1993a, 1993b, 1998; Alencar, 1998; Hamada \& McCreadie, 1999). Trabalhos taxonômicos envolvendo espécies da Amazônia Central foram realizados por Cerqueira \& Nunes de Mello (1967, 1968), PyDaniel (1983) e Hamada \& Adler (1998a; 1998b; 1999).

A nomenclatura de Simuliidae nos niveis genérico e subgenérico é controversa na região Neotropical no momento (Coscarón, 1990; Crosskey \& Howard, 1997; Py-Daniel \& Sampaio, 1994). Essa situação gera confusão para pessoas não informadas sobre a taxonomia do grupo, principalmente quanto à utilização de nomes diferentes, que se aplicam, em realidade, a uma única entidade especifica. Py-Daniel \& Sampaio (1994) sugerem que todos os subgêneros Neotropicais de Simulium sejam elevados à categoria genérica. Entretanto, como este gênero é cosmopolita e estes autores não o revisaram amplamente, nós preferimos assumir uma posição conservadora, seguindo a última lista mundial de espécie para Simuliidae de Crosskey \& Howard (1997), assim como diversos pesquisadores que trabalham com o grupo na região Neotropical (e.g. Coscarón et al., 1999; Miranda Esquivel \& Muñoz de Hoyos, 1995; Moreira \& Sato, 1996; Grillet \& Barreras, 1997; Shelley et al., 1997). Há grande probabilidade de Simulium não ser monofilético, entretanto, uma revisão criteriosa desse gênero é necessária antes de se propor mudanças nomenclaturais, que possam gerar outros grupos parafiléticos, além de promover instabilidade taxonômica.

Nove espécies de Simuliidae (Simulium cauchense Floch \& Abonnenc, Simulium daltanhani Hamada \& Adler, Simulium goeldii Cerqueira \& Nunes de Mello, Simulium maroniense Floch \& Abonnenc, Simulium perflavum Roubaud, Simulium quadrifidum Lutz, Simulium rorotaense Floch \& Abonnenc, Simulium trombetense Hamada, Py-Daniel \& Adler e Simulium "6-B1") foram registradas anteriormente nos igarapés locais (Cerqueira \& Nunes de Mello, 1968; Dellome Filho, 1978; Hamada, 1993a; Hamada \& Adler, 1998a, 1998b). Fêmeas de Simulium argentiscutum Shelley \& Luna Dias têm sido coletadas na regiăo, picando seres humanos (Py-Daniel, 1983), mas até o momento não se tem registro de seus imaturos nos cursos d'água na área de estudo.

O presente trabalho tem como objetivo fornecer dados bionômicos, de distribuição e chaves de identificação das espécies de Simuliidae coletadas até o momento na Amazônia Central, servindo de subsídios para futuros trabalhos de ecologia envolvendo comunidades de insetos aquáticos na região.

\section{MATERIAL E MÉTODOS}

A área de estudo abrangeu igarapés presentes em seis municipios no estado do Amazonas: Manaus, 
Presidente Figueiredo, Manacapuru, Careiro da Várzea, Novo Airão e Itacoatiara. Essa área é denominada no presente trabalho como Amazônia Central.

A amostragem foi realizada no período de março a agosto de 1996, entretanto, coletas adicionais foram realizadas em 1997 e 1998 para a obtenção de espécimes para estudos taxonômicos. Foram avaliados oito parâmetros físico-químicos dos cursos d'água para a caracterização do criadouro de cada espécie, no caso de espécies raras a caracterização se torna limitada devido ao baixo número de locais avaliados. A largura, profundidade, velocidade e vazão da água foi estimada utilizando-se uma régua de metal (Craig, 1987). O $\mathrm{pH}$ foi avaliado com um pHmetro Cole Parmer (pHTestr 2) e a temperatura da água foi medida com um termômetro de mercúrio de escala de $0-50^{\circ} \mathrm{C}$. A média dos parâmetros físico-químicos do habitat foram comparadas por meio do teste Kruskall-Wallis e Dunn (Zar, 1996). A presença de represamento d'água, a montante do local amostrado e o tipo de vegetação (floresta, campina, área desmatada/aberta) circundando a área estudada foi avaliada visualmente, e categorizadas nominalmente. Esses parâmetro nominais foram comparados por meio de um teste $G$, ajustado com a correção de William (Zar, 1996).

As figuras que ilustram esse trabalho foram realizados com o auxilio de uma câmara clara, acoplada ao microscópio estereoscópico ou óptico, dependendo do tamanho da estrutura a ser ilustrada. Fotografias foram realizadas por meio de uma câmara Nikon, acoplada à ocular do microscópio ótico. As espécies foram identificadas utilizando-se os trabalhos de Cerqueira \& Nunes de Mello (1968); Coscarón (1990); Coscarón et al. (1996); Dellome Filho (1978); Floch \& Abonnenc (1946); Hamada \& Adler (1998b) e Shelley \& Luna Dias (1980). Espécies do grupo Simulium perflavum foram identificadas em nivel citotaxonômico (Hamada \& Adler, 1999), utilizando-se a técnica de Rothfels \& Dunbar (1953).

Amostras de todas as espécies examinadas estão depositadas na Coleção de Invertebrados do Instituto Nacional de Pesquisas da Amazônia (INPA), Manaus, AM.

\section{RESULTADOS}

Onze espécies de Simuliidae foram coletadas, na fase de larva e/ou pupa, em um total de 79 locais amostrados nos seis municipios (Tab.1). Simulium argentiscutum foi coletada apenas no estágio adulto, de fêmeas picando seres humanos durante o periodo de amostragem, no município de Itacoatiara. Dessa forma, larvas, pupas e machos dessa espécie, utilizadas na confecção das chaves de identificações são provenientes da Coleção de Simuliidae do Instituto Oswaldo Cruz, Rio de Janeiro (pupas) e de coletas no rio Mamoré (larvas, pupas e adultos Mirante da Cachoeira, Guajará-Mirim, Rondônia, Brasil, IX/1999, Cols. N. Hamada \& U.C.Barbosa).

As espécies coletadas nos 
Tabela 1. Locais de coleta de Simulium spp. (Diptera: Simuliidae) na Amazónia Central, Brasil, Março a Agosto de 1996.

\begin{tabular}{|c|c|c|c|c|c|}
\hline $\begin{array}{c}\text { Número } \\
\text { de } \\
\text { coletas }\end{array}$ & $\begin{array}{c}\text { Nủmero } \\
\text { do } \\
\text { igarapé }\end{array}$ & Locais de coleta & Data & Coletores & $\begin{array}{l}\text { Espécies } \\
\text { coletadas: }\end{array}$ \\
\hline
\end{tabular}

CAREIRO DA VARZEA

\begin{tabular}{|c|c|c|c|c|c|}
\hline 1 & 1 & $\begin{array}{l}\text { Ramal do Cobra, estrada do Purupurú. } \\
\text { Segunda ponte de madeira } 03^{\circ} 23^{\prime} S ; 59^{\circ} 38^{\prime} \mathrm{W} \text {. }\end{array}$ & 08.03 .96 & $\mathrm{NH}$ & $1,3,6$ \\
\hline 2 & 2 & $\begin{array}{l}\text { Ramal do Cobra, estrada do Purupurú. } \\
\text { Primeira ponte de madeira. } 03^{\circ} 23^{\prime} \mathrm{S} ; 59^{\circ} 38^{\prime} \mathrm{W} \text {. }\end{array}$ & 08.03 .96 & $\mathrm{NH}$ & $1,3,6,7$ \\
\hline \multicolumn{6}{|c|}{ ITACOATIARA } \\
\hline 3 & 70 & $\begin{array}{l}\text { Rod. AM010 Fazenda Aruanã, igarapé próximo } \\
\text { ao curral. } 03^{\circ} 00^{\prime} S ; 59^{\circ} 49^{\prime} \mathrm{W} \text {. }\end{array}$ & 27.05 .96 & $\mathrm{NH} ; \mathrm{LA}$ & 1,7 \\
\hline 4 & 71 & $\begin{array}{l}\text { Rod. AM010 Fazenda Aruanã, próximo a } \\
\text { plantaçăo de pupunha. } 03^{\circ} 00^{\prime} S: 59^{\circ} 49^{\prime} \mathrm{W} \text {. }\end{array}$ & 28.05 .96 & $\mathrm{NH} ; \mathrm{LA}$ & 3,4 \\
\hline 5 & 72 & $\begin{array}{l}\text { Rod, AM010 Ramal do Mirapocuzinho, sitio de } \\
\text { D. Belinha. } 02^{\circ} 58^{\prime} S ; 59^{\circ} 04^{\prime} \mathrm{W} \text {. }\end{array}$ & 28.05 .96 & NH:LA & 7 \\
\hline 6 & 73 & $\begin{array}{l}\text { Estrada para Itapiranga, Madereira Mil, } \\
03^{\circ} 05^{\prime} \mathrm{S} ; 59^{\circ} 60^{\prime} \mathrm{W} \text {. }\end{array}$ & 28.05 .96 & NH:LA & 5.7 \\
\hline 7 & 74 & $\begin{array}{l}\text { Rod. AM010, ramal Săo Geraldo, próximo à } \\
\text { fazenda Aruană. } 03^{\circ} 00^{\prime} \text {; } 58^{\circ} 49^{\prime} \mathrm{W} \text {. }\end{array}$ & 28.05 .96 & $\mathrm{NH}, \mathrm{LA}$ & 7 \\
\hline 8 & 75 & $\begin{array}{l}\text { Rod. AM010, ramal Nova vida, próximo à } \\
\text { fazenda Aruanã. } 03^{\circ} 00^{\prime} S ; 58^{\circ} 49^{\prime} \mathrm{W} \text {. }\end{array}$ & 28.05 .96 & $N H_{i} L A$ & 5,7 \\
\hline 9 & 76 & $\begin{array}{l}\text { Rod. AM010, ramal Nova vida, próximo à } \\
\text { fazenda Aruaná. } 03^{\circ} 00^{\circ} \mathrm{S} ; 58^{\circ} 49^{\prime} \mathrm{W} \text {. }\end{array}$ & 28.05 .96 & NH:LA & 7 \\
\hline 10 & 77 & $\begin{array}{l}\text { Rod. AM010, ramal Nova vida } \mathrm{km} 8 \text {, próximo à } \\
\text { fazenda Aruană. } 03^{\circ} 00^{\prime} \mathrm{S} ; 58^{\circ} 49^{\prime} \mathrm{W} \text {. }\end{array}$ & 28.05 .96 & NH:LA & 1.3 .6 \\
\hline 11 & 78 & $\begin{array}{l}\text { Rod. AM010, igarapé próximo à lagoa do Vale } \\
\text { dos Inocentes, fazenda Aruanā. } 03^{\circ} 00^{\circ} \text { : } \\
58^{\circ} 49^{\prime} \mathrm{W} \text {. }\end{array}$ & 29.05 .96 & $\mathrm{NH} ; \mathrm{LA}$ & 2,3 \\
\hline 12 & 79 & $\begin{array}{l}\text { Rod. AM010, depois da fazenda Pună } 02^{\circ} 55^{\prime} \mathrm{S} \text {; } \\
58^{\circ} 57^{\prime} \mathrm{W} \text {. }\end{array}$ & 29.05 .96 & $N H: L A$ & $6,7,8$ \\
\hline \multicolumn{6}{|c|}{ MANAUS } \\
\hline 13 & 3 & $\begin{array}{l}\text { Rod. AM010 km 51, Igarapé - km 8, CiGS, } \\
02^{\circ} 45^{\prime} S ; 59^{\circ} 51^{\prime} \mathrm{W} \text {. }\end{array}$ & 18.03 .96 & $\mathrm{NH}$ & $1,5,7$ \\
\hline 14 & 5 & $\begin{array}{l}\text { Rod. AM010 km 24, Igarapé } 1000 \text {, Reserva } \\
\text { Ducke, } 02^{\circ} 57^{\prime} \mathrm{S}: 59^{\circ} 57^{\prime} \mathrm{W} \text {. }\end{array}$ & $19,03,96$ & NH & $3,4,8$ \\
\hline 15 & 6 & $\begin{array}{l}\text { Rod. AM010 km 24, Igarapé } 700 \text {, Reserva } \\
\text { Ducke. } 02^{\circ} 57^{\prime} \mathrm{S}: 59^{\circ} 57^{\prime} \mathrm{W} \text {. }\end{array}$ & 19.03 .96 & $\mathrm{NH}$ & 3,4 \\
\hline 16 & 7 & $\begin{array}{l}\text { Rod. AM010 km 24, Igarapé I. Reserva Ducke. } \\
02^{\circ} 57^{\prime} \mathrm{S} ; 59^{\circ} 57^{\prime} \mathrm{W} \text {. }\end{array}$ & 19.03 .96 & $\mathrm{NH}$ & 3. 4,8 \\
\hline
\end{tabular}


Tabela 1. Continuação

Rod. AM010 km 24, Igarapé Acará Reserva Ducke. $02^{\circ} 57^{\prime} \mathrm{S} ; 59^{\circ} 57^{\prime} \mathrm{W}$

Rod. AM010 km 24, Igarapé Barro Banco, Reserva Ducke. 02 $34^{\prime} \mathrm{S}$ $60^{\circ} 07^{\prime} \mathrm{W}$.

Cidade de Deus, Manaus, antes da entrada para o Sabia I - Reserva Ducke. $03^{\circ} 00^{\prime} S$; $59^{\circ} 56^{\prime} \mathrm{W}$,

Ramal entre Puraquequara e Jorge Teixeira, Manaus. $03^{\circ} 02^{\prime} S ; 59^{\circ} 53^{\prime} \mathrm{W}$.

Ramal entre Puraquequara e Jorge Teixeira, Manaus. $03^{\circ} 02^{\prime} \mathrm{S} ; 59^{\circ} 53^{\prime} \mathrm{W}$.

Ramal entre Puraquequara e Jorge Teixeira, Manaus. $03^{\circ} 02$ 'S; $59^{\circ} 53^{\prime} \mathrm{W}$.

Ramal entre Puraquequara e Jorge Teixeira, Manaus, $03^{\circ} 02^{\prime} \mathrm{S} ; 59^{\circ} 53^{\prime} \mathrm{W}$.

Antes da entrada do ramal para a Praia Dourada (Igarapé Tarumã), Manaus. $03^{\circ} 01^{\prime} \mathrm{S} ; 60^{\circ} 04^{\prime} \mathrm{W}$.

Cachoeira das Almas, Manaus. $03^{\circ} 00^{\prime} \mathrm{S}$ : $60^{\circ} 03^{\prime} \mathrm{W}$

Vivenda do Pontal, Manaus. 02057'S: $60^{\circ} 04^{\prime} \mathrm{W}$.

Ramal em frente ao sítio Lobato,

próximo à entrada da Vivenda Verde, Manaus. $02^{\circ} 58^{\prime} \mathrm{S} ; 60^{\circ} 04^{\prime} \mathrm{W}$.

Ramal do Riacho Ecológico, entrada para a Trigolar, Chácara Sảo

Sebastião, Manaus. $02^{\circ} 58^{\prime} \mathrm{S}$; $60^{\circ} 03^{\prime} \mathrm{W}$.

Rod. AM010 km 24, Igarapé Barro Banco, abalxo da piscina, área aberta, Reserva Ducke. $02^{\circ} 57^{\prime} \mathrm{S} ; 59^{\circ} 57^{\prime} \mathrm{W}$.

Rod. AM010 km 30, Cachoeira no lgarapé Leăo. $02^{\circ} 49^{\prime}$ S; $59^{\circ} 58^{\prime} W$.

Rod. AM 0101 igarapé depois do Igarapé Água Branca, no ramal Água Branca I. $02^{\circ} 49^{\prime} \mathrm{S} ; 59^{\circ} 58^{\prime} \mathrm{W}$.

Rod. AM $0102{ }^{\circ}$ igarapé depois do 54 Igarapé Água Branca, no ramal Agua Branca I. $02^{\circ} 49^{\prime}$ S; $59^{\circ} 58^{\prime} \mathrm{W}$.

Rod. AM $0104^{2}$.igarapé depois do 55 Igarapé Água Branca, no ramal Água Branca I. $02^{\circ} 49^{\prime} \mathrm{S} ; 59^{\circ} 58^{\prime} \mathrm{W}$.

Rod. AM $0107^{2}$.igarapé depois do Igarapé Água Branca, no ramal Água Branca I. $02^{\circ} 49^{\prime} S$; $59^{\circ} 58^{\prime} \mathrm{W}$.

HW AM $0106 \%$. igarapé depois do Branca I. $02^{\circ} 49^{\prime} \mathrm{S} ; 59^{\circ} 58^{\prime} \mathrm{W}$

19.03 .96

$\mathrm{NH}$

$3,4,8$

01.04 .96

NH; FFXF

3,4

01.04 .96 NH: FFXF $\quad 1,5,7,12$

25.04 .96

$\mathrm{NH}$

25.04 .96

$\mathrm{NH}$

1,4

25.04 .96

$\mathrm{NH}$

25.04 .96

NH

1

02.05 .96

$\mathrm{NH}$

7

02.05 .96

$\mathrm{NH}$

1,7

02.05 .96

$\mathrm{NH}$

7

02.05 .96

$\mathrm{NH}$

3,5

02.05 .96

$\mathrm{NH}$

3. 4

09.05 .96

$3,4,8$

09.05 .96 
Tabela 1. Continuação

\begin{tabular}{|c|c|c|c|c|c|}
\hline 37 & 61 & $\begin{array}{l}\text { ZF3 km } 41 \text {, Reserva } 1501 \text { INPA/PDBFF, } \\
\text { igarapé na linha J, próximo ao } \\
\text { acampamento } 02^{\circ} 25^{\prime} \mathrm{S} ; 59^{\circ} 43^{\prime} \mathrm{W} \text {. }\end{array}$ & 20.05 .96 & $\mathrm{NH}$ & $3,4,8$ \\
\hline 38 & 62 & $\begin{array}{l}\text { ZF3 km 41, Reserva } 1501 \text { INPA/PDBFF, } \\
\text { igarapé formado pela junçẫo de dois } \\
\text { pequenos. } 02^{\circ} 25^{\prime} \mathrm{S} ; 59^{\circ} 43^{\prime} \mathrm{W}\end{array}$ & 20.05 .96 & $\mathrm{NH}$ & 8,12 \\
\hline 39 & 63 & $\begin{array}{l}\text { ZF3 km } 41 \text {, Reserva } 1501 \text { INPA/PDBFF, } \\
02^{\circ} 25^{\prime} \mathrm{S} ; 59^{\circ} 43^{\prime} \mathrm{W} \text {. }\end{array}$ & 21.05 .96 & NH & 4 \\
\hline 40 & 64 & $\begin{array}{l}\text { ZF3 km } 41 \text {, Reserva } 1501 \text { INPA/PDBFF. } \\
02^{\circ} 25^{\prime} \mathrm{S} ; 59^{\circ} 43^{\prime} \mathrm{W} \text {. }\end{array}$ & 21.05 .96 & $\mathrm{NH}$ & 12 \\
\hline 41 & 65 & $\begin{array}{l}\text { Rod. AM010 km } 30 \text {, ramal Água Branca II, } \\
\text { Igarapé Matrinxã, Green-Park. } 02^{\circ} 51^{\prime} \mathrm{S} ; 59^{\circ} \\
51^{\prime} \text { W. }\end{array}$ & 23.05 .96 & $\mathrm{NH}$ & 1,2 \\
\hline 42 & 66 & $\begin{array}{l}\text { Rod. AM010 km } 30 \text {, ramal Água Branca II, } \\
\text { Igarapé Puraquequara, Green-Park. } 02^{\circ} 51^{\prime} \mathrm{S} \text {; } \\
59^{\circ} 51^{\prime} \mathrm{W} \text {. }\end{array}$ & 23.05 .96 & $\mathrm{NH}$ & 2,4 \\
\hline 43 & 67 & $\begin{array}{l}\text { Rod. AM010 km 30, Ramal Água Branca II, } \\
\text { pequena estrada à esquerda do ramal para } \\
\text { o Green-Park. } 02^{\circ} 51^{\prime} \mathrm{S} ; 59^{\circ} 51^{\prime} \mathrm{W} \text {. }\end{array}$ & 23.05 .96 & $\mathrm{NH}$ & $\begin{array}{c}1,2 \\
12\end{array}$ \\
\hline 44 & 68 & $\begin{array}{l}\text { Rod. AM010 km } 30 \text {, Ramal Água Branca II. } \\
\text { pequeno igarapé no Aquário Prestige. } \\
02^{\circ} 51^{\prime} \mathrm{S} ; 59^{\circ} 51^{\prime} \mathrm{W} \text {. }\end{array}$ & 23.05 .96 & $\mathrm{NH}$ & 1,7 \\
\hline 45 & 80 & $\begin{array}{l}\text { Reserva Ducke, Igarapé Limpo, Sabiá I. } \\
03^{\circ} 00^{\prime} \mathrm{S} ; 59^{\circ} 56^{\prime} \mathrm{W} \text {. }\end{array}$ & 30.05 .96 & $\mathrm{NH}$ & 3,4 \\
\hline 46 & 81 & $\begin{array}{l}\text { Ramal para Santa Etelvina, próximo ao sitio } \\
\text { Fortaleza, igarapé sedimentado. } 02^{\circ} 59^{\prime} \mathrm{S} \text {; } \\
59^{\circ} 58^{\prime} \mathrm{W} \text {. }\end{array}$ & 30.05 .96 & $\mathrm{NH}$ & 1 \\
\hline 47 & 82 & $\begin{array}{l}\text { Rod, AM010, ramal próximo ao posto } \\
\text { rodoviário, tributário do Igarapé Acará. } \\
02^{\circ} 57^{\prime} \mathrm{S} ; 59^{\circ} 59^{\prime} \mathrm{W} \text {. }\end{array}$ & 30.05 .96 & $\mathrm{NH}$ & 1 \\
\hline & & MANACAPURU & & & \\
\hline 48 & 9 & $\begin{array}{l}\text { Rod. AM352 estrada para Novo Alrão } \\
\text { próximo à estrada para Manacapuru. } \\
03^{\circ} 13^{\prime} S: 60^{\circ} 39^{\prime} \mathrm{W} \text {. }\end{array}$ & 23.03 .96 & $\mathrm{NH}$ & 7 \\
\hline 49 & 10 & $\begin{array}{l}\text { Rod. AM352 estrada para Novo Airão- } \\
\text { depois do ponto \#9. } 03^{\circ} 13^{\prime} \text { S: } 60^{\circ} 39^{\prime} W \text {. }\end{array}$ & 23.03 .96 & $\mathrm{NH}$ & 7 \\
\hline 50 & 11 & $\begin{array}{l}\text { Rod. AM352 estrada para Novo } \\
\text { Airãodepois do ponto \#10.0 } 03^{\circ} 13^{\prime} \text { S; } \\
60^{\circ} 39^{\prime} \mathrm{W} \text {. }\end{array}$ & 23.03 .96 & $\mathrm{NH}$ & 7 \\
\hline 51 & 12 & $\begin{array}{l}\text { Rod. AM352 estrada para Novo Airäo } \\
\text { depois do ponto \#11. } 03^{\circ} 13^{\prime} \mathrm{S} ; 60^{\circ} 39^{\prime} \mathrm{W} \text {. }\end{array}$ & $23,03,96$ & $\mathrm{NH}$ & $1,2,3$ \\
\hline \multicolumn{6}{|c|}{ NOVO AIRĀO } \\
\hline 52 & 14 & $\begin{array}{l}\text { Rod. AM352 estrada para Novo Airão. } \\
03^{\circ} 13^{\prime} S ; 60^{\circ} 40^{\prime} \mathrm{W} \text {. }\end{array}$ & 23.03 .96 & $\mathrm{NH}$ & 1,6 \\
\hline 53 & 15 & $\begin{array}{l}\text { Rod, } A M 352 \text { estrada para Novo Airăo } \\
\text { depois do ponto } \# 14.03^{\circ} 13^{\prime} S ; 60^{\circ} 40^{\prime} \mathrm{W} \text {. }\end{array}$ & $23,03.96$ & $\mathrm{NH}$ & 1,6 \\
\hline
\end{tabular}


Rod. BR174 km 107, pequeno igarapé na fazenda Santa Claudia, próximo à fonte. $02^{\circ} 02^{\prime} \mathrm{S} ; 60^{\circ} 00^{\prime} \mathrm{W}$.

$55 \quad 17 \quad \begin{aligned} & \text { Rod. BR174 } \mathrm{km} \mathrm{107,} \mathrm{Cachoeira} \mathrm{da} \\ & \text { fazenda Santa Claudia. 02 02 } 02^{\prime} \mathrm{S} ;\end{aligned}$ $60^{\circ} 00^{\prime} \mathrm{W}$.

56

18 Cachoeira da Onça. $02^{\circ} 02^{\prime}$ S; $60^{\circ} 02^{\prime} W$.

Rod. AM210 km 10, para Balbina. $02^{\circ} 50^{\prime} \mathrm{S}$; $60^{\circ} 10^{\prime} \mathrm{W}$.

\section{Estrada năo pavimentada entre} Presidente Figueiredo e estrada para
Balbina, próximo ao cemitério. $02^{\circ} 02^{\prime} \mathrm{S}$; $60^{\circ} 39^{\prime} \mathrm{W}$.

Rod. BR174 km 134, ramal para a $01^{\circ} 49^{\prime} \mathrm{S} ; 60^{\circ} 04^{\prime} \mathrm{W}$.

Rod. BR174 km 117, pequeno tributário

da margem esquerda do rio Urubuí, abaixo da Cachoeira Iracema. $01^{\circ \circ 9}$ 's; $60^{\circ} 03^{\prime} \mathrm{W}$.

Rod. BR174 km 117, tributário do rio

Urubui, ponte de madeira no ramal para Cachoeira Iracema. 01059'S: 60003'W.

Rod. BR174 km 113, tributário do igarapé Lajes, balneário do Sr Almir. 0159'S; $60^{\circ} 01^{\prime} \mathrm{W}$.

Rod. BR174 km 108, tributário do rio

Urubuí, $200 \mathrm{~m}$ da entrada para tanques de piscicultura abandonados. $02^{\circ} 01^{\prime} \mathrm{S}: 60^{\circ} 02^{\prime} \mathrm{W}$.

Cachoeira Orquídea, Igarapé do Veado. $02^{\circ} 02^{\prime} \mathrm{S}$; $60^{\circ} 39^{\prime} \mathrm{W}$.

Rod. BR174 km 97, Cachoeira da

SUFRAMA, tríbutário da margem direita do rio Urubu. $02^{\circ} 09^{\prime} S ; 59^{\circ} 57^{\prime} \mathrm{W}$.

Rod. BR174 km 109, Cachoeira

Cristalina, 45 minutos de caminhada da casa do Sr. Nezinho. $02^{\circ} 28^{\prime} \mathrm{S}$; $60^{\circ} 01^{\prime} \mathrm{W}$.

Rod. AM210 km 6, pequeno igarapé 6730 acima da caverna Maruaga. $02^{\circ} 02^{\prime}$ S $59^{\circ} 58^{\prime} \mathrm{W}$.

Rod. AM210 km 6, pequeno igarapé, 6831 próximo ao igarapé \#30.02\%2's; $59^{\circ} 58^{\prime} \mathrm{W}$.

$69 \quad 32$ Rod. AM210 km 12, Cachoeira Santuário. $02^{\circ} 03^{\prime} \mathrm{S} ; 59^{\circ} 55^{\prime} \mathrm{W}$.

Rod. AM210 km 20, Igarapé da Onça. $02^{\circ} 02^{\prime}$ S: $59^{\circ} 50^{\prime} \mathrm{W}$.

Rod. AM210 km 58, Igarapé Pedra
28.03 .96

25.03 .96

$\mathrm{NH}$;

FFXF

4. 8

25.03 .96

$\mathrm{NH}:$

FFXF

3,8

25.03 .96

$\mathrm{NH}$ :

FFXF

$2,3,7,8$

25.03 .96

$\mathrm{NH}$;

FFXF

3

25.03 .96

$\mathrm{NH}$;

FFXF

5, 7

26.03.96

$\mathrm{NH}$;

FFXF

$2,10,11$

26.03 .96

$\mathrm{NH}$;

FFXF

4

26.03 .96

$\mathrm{NH}$;

FFXF

$3,4,8,9$

26.03 .96

$\mathrm{NH}$ :

FFXF

4. 9

26.03 .96

$\mathrm{NH}$;

FFXF

$1,7,8,9$

27.03 .96

$\mathrm{NH}$;

FFXF

3. 4,8

27.03 .96

$\mathrm{NH}$;

FFXF

4. 8

28.03 .96

$\mathrm{NH}$ :

FFXF

8

28.03 .96

$\mathrm{NH}$;

FFXF

1, 7, 8

28.03 .96

$\mathrm{NH}$ :

FFXF

$3,4,7,8$

$\mathrm{NH}$;

FFXF

$1,3,9$

$\mathrm{NH}$;

FFXF

3. 8,9

29.03 .96

$\mathrm{NH}$;

FFXF

$3,4,8$ 
Tabela 1. Continuação

\begin{tabular}{|c|c|c|c|c|c|}
\hline 72 & 35 & $\begin{array}{l}\text { Rod. AM210 km } 58 \text {, pequeno igarapé } \\
\text { próximo ao igarapé Pedra Furada. } \\
01^{\circ} 52^{\prime} \mathrm{S} ; 59^{\circ} 58^{\prime} \mathrm{W} \text {. }\end{array}$ & 29.03 .96 & $\begin{array}{l}\mathrm{NH}_{i} \\
\mathrm{FFXF}\end{array}$ & $3,4,8$ \\
\hline 73 & 36 & $\begin{array}{l}\text { UHE Balbina, ramal para Cachoeira } \\
\text { Morena } 02^{\circ} 05^{\prime} \mathrm{S} ; 59^{\circ} 20^{\prime} \mathrm{W} \text {. }\end{array}$ & 29.03 .96 & $\begin{array}{l}\mathrm{NH} \\
\text { FFXF }\end{array}$ & 3,4 \\
\hline 74 & 37 & $\begin{array}{l}\text { Rod. AM210, ramal do } \mathrm{km} 17 \text {, Cachoeira } \\
\text { Maricota. } 02^{\circ} 02^{\prime} \mathrm{S} ; 59^{\circ} 52^{\prime} \mathrm{W} \text {. }\end{array}$ & 29.03 .96 & $\begin{array}{l}\mathrm{NH} ; \\
\mathrm{FFXF}\end{array}$ & 8 \\
\hline 75 & 38 & $\begin{array}{l}\text { Rod. AM210, ramal do } \mathrm{km} 17 \text {, pequeno } \\
\text { igarapé próximo à Cachoeira Maricota. } \\
02^{\circ} 02^{\prime} S ; 59^{\circ} 52^{\prime} \mathrm{W} \text {. }\end{array}$ & 29.03 .96 & $\begin{array}{l}\mathrm{NH} \\
\mathrm{FFXF}\end{array}$ & 3,8 \\
\hline 76 & 39 & $\begin{array}{l}\text { Rod. AM210, ramal do } \mathrm{km} 17,10 \text {. igarapé } \\
\text { que cruza o ramal. } 02^{\circ} 02^{\prime} \mathrm{S} ; 59^{\circ} 52^{\prime} \mathrm{W} \text {. }\end{array}$ & 29.03 .96 & $\begin{array}{l}\mathrm{NH} \\
\mathrm{FFXF}\end{array}$ & 4 \\
\hline 77 & 118 & $\begin{array}{l}\text { UHE Balbina, ramal para Cahoeira } \\
\text { Morena, Cachoeira do Boto, } 02^{\circ} 05^{\prime} \mathrm{S} \text {; } \\
59^{\circ} 20^{\prime} \mathrm{W} \text {. }\end{array}$ & 10.08 .96 & $\begin{array}{l}\mathrm{NH} ; \\
\text { FFXF }\end{array}$ & $1,2,8,9$ \\
\hline 78 & 119 & $\begin{array}{l}\text { UHE Balbina, ramal para Cachoeira } \\
\text { Morena, fazenda Lindo Amanhecer. } \\
02^{\circ} 05^{\prime} \mathrm{S} ; 59^{\circ} 20^{\prime} \mathrm{W} \text {. }\end{array}$ & 10.08 .96 & $\begin{array}{l}\mathrm{NH} ; \\
\text { FFXF }\end{array}$ & 4,8 \\
\hline 79 & 120 & $\begin{array}{l}\text { UHE Balbina, ramal para Cachoeira } \\
\text { Morena, segunda ponte de madeira, } \\
\text { depois do ponto n. } 119.02^{\circ} 05^{\prime} S ; 59^{\circ} 20^{\prime} W \text {, }\end{array}$ & $10,08,96$ & $\begin{array}{l}\mathrm{NH}_{i} \\
\mathrm{FFXF}\end{array}$ & $1,4,8$ \\
\hline
\end{tabular}

Nota: Rod. = rodovia; $\mathrm{BR}=$ rodovia federal; $\mathrm{AM}=$ rodovia do estado do Amazonas; UHE = Usina hidroelétrica; INPA = Instituto Nacional de Pesquisas da Amazônia; PDBFF = Projeto Dinâmica Biológica de Fragmentos Florestais. Data $=$ dia/mês/ano; Coletor, FFXF $=$ Francisco F.Xavier Filho, $L A=$ Luis Aquino, NH $=$ Neusa Hamada.

*Espécies coletadas nos igarapés: 1 = Simulium quadrifidum; 2 = Simulium cauchense; $3=$ Simulium goeldii; 4 = Simulium "6-B1"; 5 = Simulium daltanhani; 6 = Simulium "A"; 7 = Simulium perflavum; 8 = Simulium rorotaense; 9 $=$ Simulium trombetense, $10=$ Simulium maroniense, $11=$ Simulium iracouboense, $12=$ larvas imaturas de $S$.

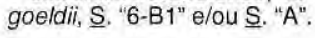

igarapés foram: $S$. cauchense, $S$, daltanhani, S. goeldii, Simulium iracouboense Floch \& Abonnenc, $S$. maroniense, $S$. perflavum, $S$. quadrifidum, $S$, rorotaense, $S$. trombetense, $S$. "6-B1" e $S$. "A". Simulium maroniense foi identificada como pertencente ao citotipo D (Hamada \& Adler, 1999).

Nas Figuras 1 e 2, apresentamos as espécies de Simuliidae coletadas nos igarapés, as condições médias (士erro padrão) e a proporção de ocorrência de acordo com os diferentes parâmetros avaliados. As espécies $S$. maroniense e $S$. iracouboense não foram incluídas nos gráficos, uma vez que ocorreram apenas em um igarapé.

Os resultados sugerem que cada espécie ocupa uma faixa característica dentro da disponibilidade de habitat, podendo, por exemplo, serem caracterizadas como espécies de floresta (S. goeldii, S. "6-B1", S. rorotaense e $S$. trombetense), de ambiente perturbado, geralmente associados a represamentos artificiais de água ( $S$. perflavum e $S$. daltanhani) ou ocupando habitats intermediários (S. quadrifidum, S. "A").

\section{Chave de identificação para machos}

1. Espécies amarelas ou laranjas... 2 1 '.Espécies negras................ 5 

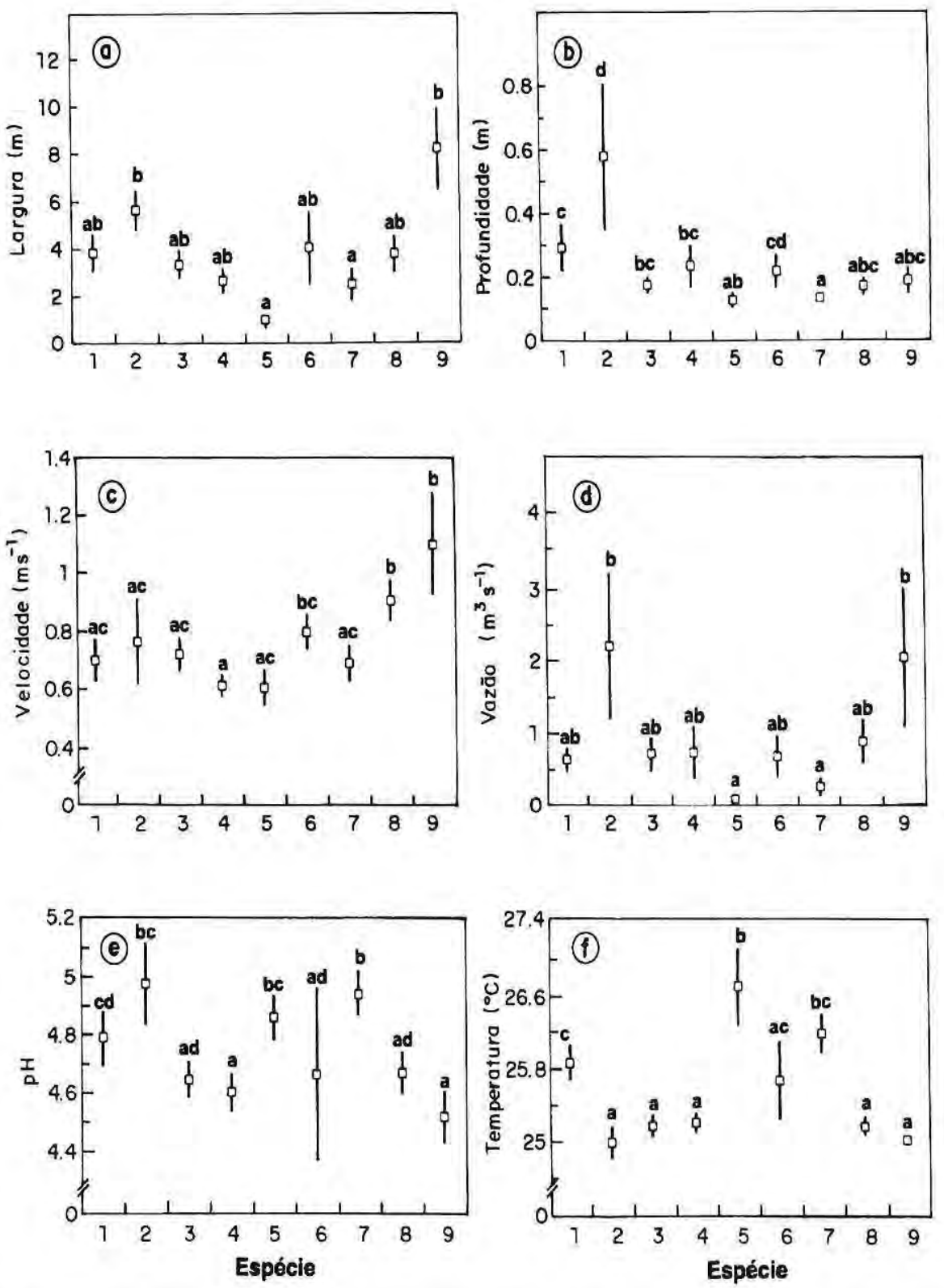

Figura 1. Valores médios dos parâmetros físico-químicos dos igarapés onde as espécies de simulideos foram coletadas na Amazônia Central, Brasil. a) largura (m); b) profundidade (m); c) velocidade $\left(\mathrm{ms}^{-1}\right)$; d) vazão $\left(\mathrm{m}^{3} \mathrm{~s}^{-1}\right)$; e) temperatura $\left({ }^{\circ} \mathrm{C}\right)$; f) $\mathrm{pH}$. Notas: espécie, $1=S$. quadrifidum, $2=S$. cauchense, $3=S$. goeldii, $4=S$. "6-B1", $5=S$. daltanhani, $6=S$. "A", 7 $=S$. perflavum, $8=S$. rorotaense, $9=S$. trombetense; barra vertical representa o erro padrão $( \pm \mathrm{EP})$. Médias seguidas com as mesmas letras não significativamente diferentes a nivel de $5 \%$ (teste de Dunn). 


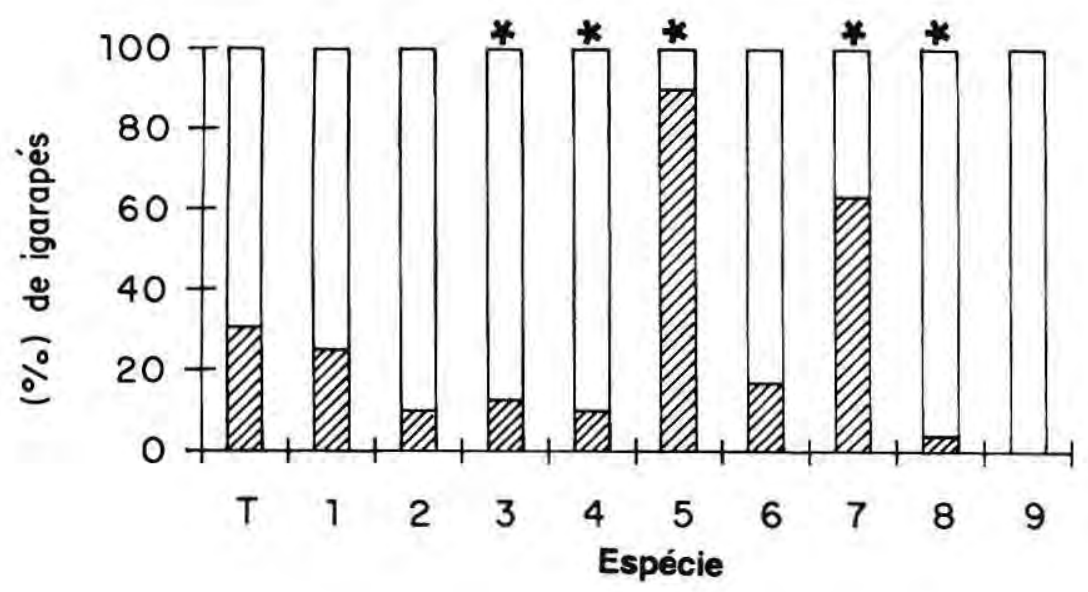

Com represamento $\square$ Sem represomento
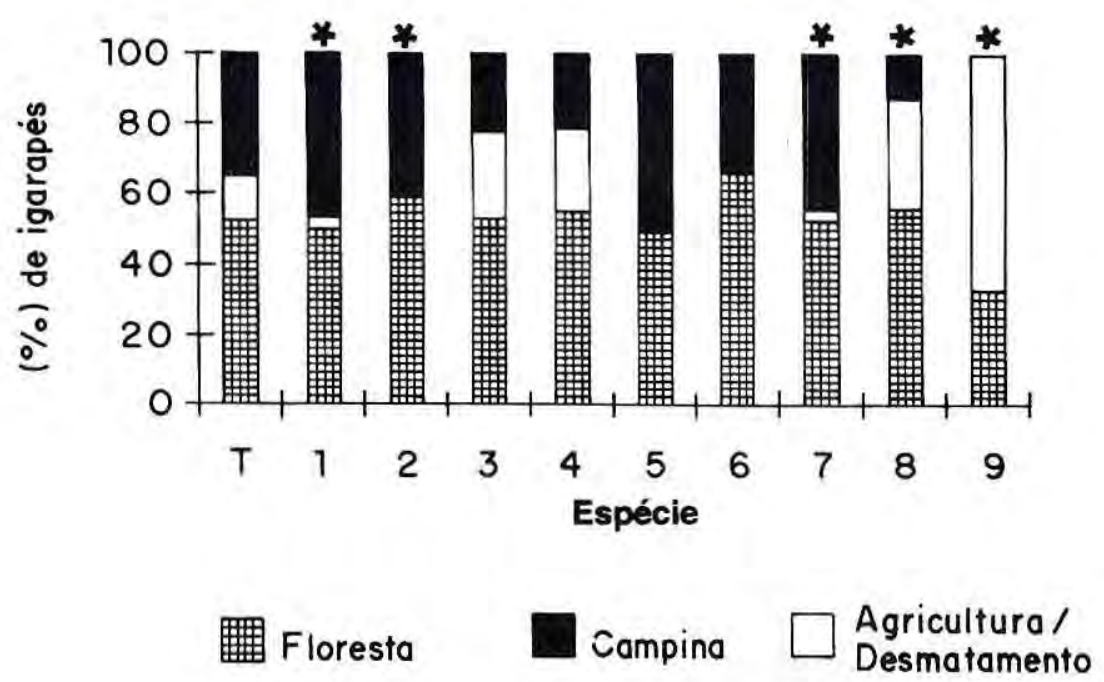

Figura 2. Distribuição de espécies de simulídeos (Diptera: Simuliidae): a) em igarapés com e sem represamento; b) e em três tipos de uso da terra na Amazônia Central, Brasil. Notas: $T=$ total de igarapés amostrados $(\mathrm{n}=79)$; espécies, $1=S$. quadrifidum; $2=S$. cauchense; $3=S$. goeldii; $4=S$. "6-BI"; $5=S$. daltanhani; $6=S$. "A"; $7=S$. perflavum $; 8=S$, rorotaense $; 9=$ $S$. trombetense). ${ }^{*} \mathrm{p}<0,05$ (teste $\mathrm{G}$, com ajuste de Williams). 
2. Escudo com 1 par de bandas submedianas prateadas (Fig. 4)

S. iracouboense

2'. Escudo sem 1 par de bandas submedianas 3

3. Antenas (Fig. 11) e pernas medianas (Fig. 13) sem regiões escuras .......................... S. perflavum

3'. Antenas (Fig. 12) e pernas medianas (Fig. 14) com regiões escuras 4

4. Todos os tergitos abdominais com manchas amarelo-alaranjadas, espécimes grandes ...... S. trombetense

4'. Nem todos os tergitos abdominais com manchas amarelo-alaranjadas, espécimes de tamanho mediano ...

$S$. maroniense e S. rorotaense

5. Escudo com 1 par de manchas submedianas prateadas (Figs. 6, 8, 10) .. 6

5'. Escudo sem manchas submedianas prateadas aparentes ........ 8

6. Manchas submedianas do escudo com área basal grossa, conectadas à banda lateral prateada (Fig.6). $S$. argentiscutum

6'. Manchas submedianas no escudo com área basal fina (Figs. 8, 10), sem conexão com a banda lateral prateada 7

7. Escudo com 1 par de manchas submedianas finas e alongadas (Fig. 8).....

S. quadrifidum

7'. Escudo com 1 par de manchas submedianas cuneiformes (Fig. 10)

S. cauchense

8. Espécimes grandes $(>2,0 \mathrm{~mm})$, distimero com 2 a 3 espinhos (Fig. 15)

S. daltanhani

8'. Espécimes pequenos $(<2,0$ $\mathrm{mm})$, distímero com 1 espinho ... S. goeldii, S. "6-B1", S. "A"

\section{Chave de identificação para fêmeas}

1-Espécies amarelas ou laranjas ....

1'- Espécies negras ..................... 5

2 - Escudo com 1 par de bandas submedianas prateadas (Fig. 3) .... S. iracouboense

2'- Escudo sem bandas submedianas 3

3 - Antena com flagelômeros basais alargados (Fig. 11), antenas e pernas medianas sem mancha escura (Fig. 13) S. perflavum

3'- Antena com flagelômeros basais não alargados (Fig. 12); antenas e pernas medianas com mancha escura (Fig. 14) 4

4 - Projeção do lobo anal, em vista lateral, 1,2 a 1,3 vezes tão longa quanto a largura basal (Fig. 16)

S. trombetense

4'- Projeção do lobo anal, em vista lateral, 1,9 vezes tão longa quanto a largura basal (Fig. 17)

S. maroniense e $S$, rorotaense

5 - Com padrão distinto no escudo (Figs. 5, 7, 9)

5 ' - Sem padrão distinto no escudo 8

6 - Escudo com 1 par de manchas cuneiformes, submedianas (Fig. 9) S. cauchense

6' - Escudo sem 1 par de manchas cuneiformes, com padrão no escudo diverso (Figs. 5, 7)

7 - Escudo negro, com 1 par de bandas longitudinais (Fig. 7), submedianas e prateadas S. quadrifidum

7' - Escudo acinzentado, com padrão do escudo como na Fig. 5 S. argentiscutum 
$8-$ Espécimes grandes $($ corpo $=$ 1,9-2,4 mm), órgão sensorial do palpo ocupando mais da metade do segmento que ocupa (Fig. 18) ......

S. daltanhani

8'- Espécimes pequenos (corpo $=1,5-1,75 \mathrm{~mm}$ ), órgão sensorial do palpo ocupando menos da metade do segmento que ocupa (Fig. 19) ..... S. "6-B1", S. "A" e S. goeldii

Chave de identificação para pupas

1 - Espécies com quatro filamentos branquiais; tubérculos cefálicos ponteagudos (Fig. 20) ..... 2

1 '- Espécies com mais de quatro filamentos branquiais; tubérculos cefálicos não ponteagudos (Figs. 2123)

2- Ramos primários dos filamentos branquiais se bifurcando mais da metade do comprimento total
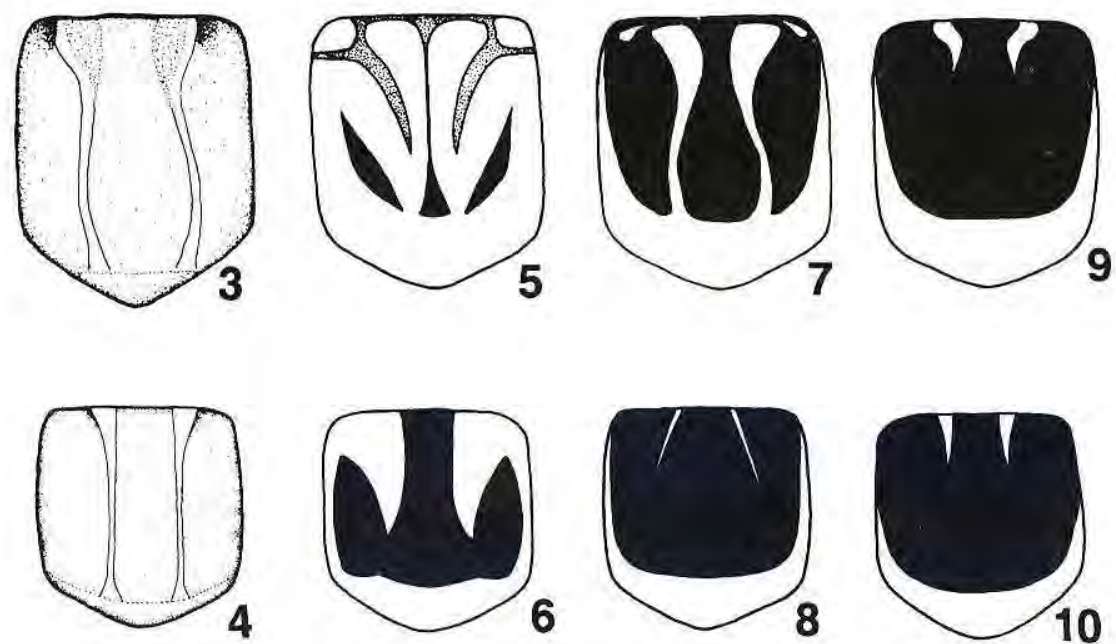

Figuras 3-10. Escudo. Simulium iracouboense fềmea (3) e macho (4). Simulium argentiscutum fềmea (5) e macho (6). Simulium quadrifidum fêmea (7) e macho (8). Simulium cauchense fêmea (9) e macho (10). 

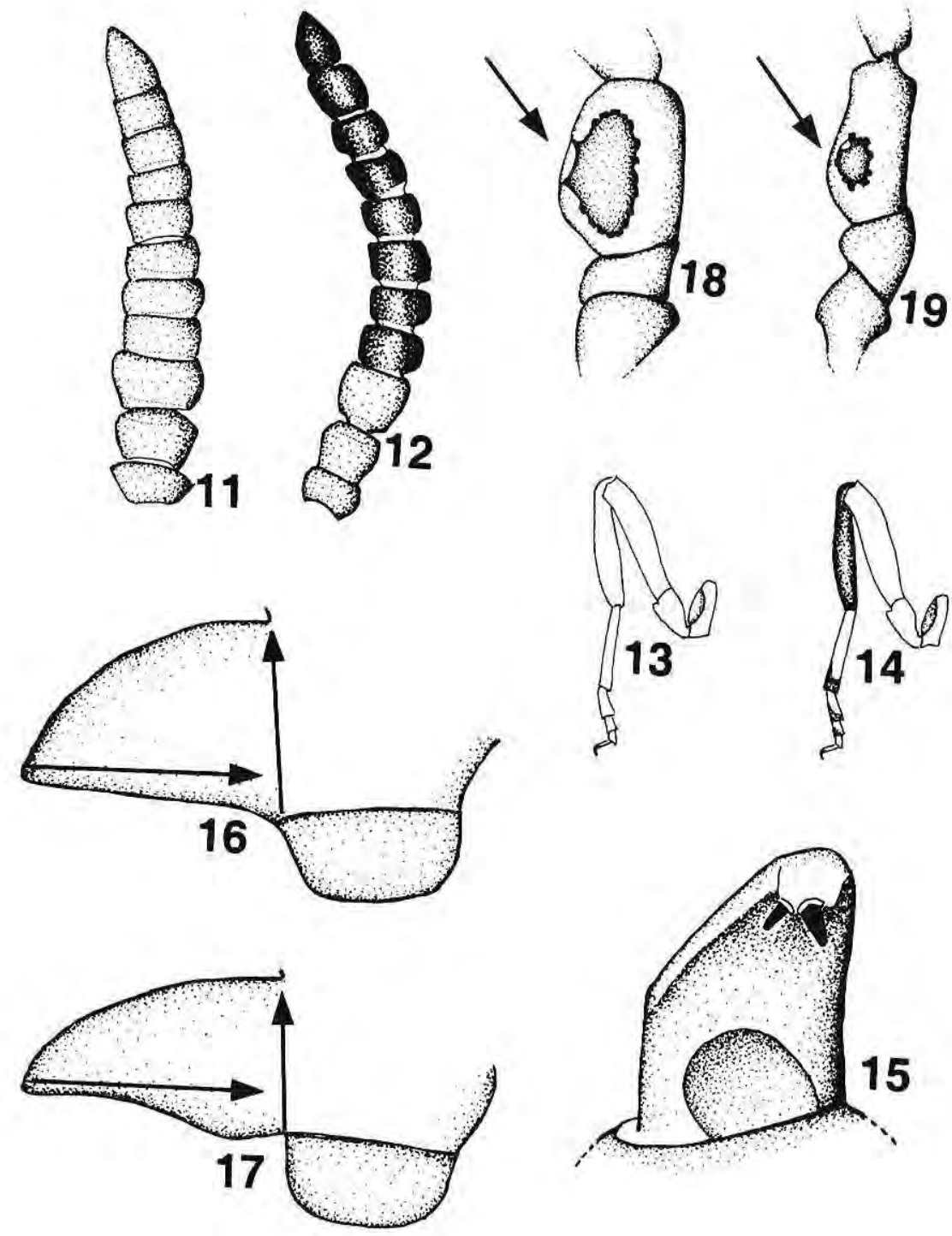

Figuras 11-19. Antena de Simulium perflavum (11) e Simulium rorotaense (12). Perna mediana de Simulium perflavum (13) e Simulium rorotaense (14). Macho de Simulium daltanhani: distímero (15). Fêmea de Simulium trombetense: lobo anal (16). Fêmea de Simulium rorotaense: lobo anal (17). Orgão sensorial no terceiro segmento do palpo da fêmea de Simulium daltanhani (18) e Simulium goeldii (19). 
(diâmetro, $\overline{\mathrm{x}}=12 \mu, \mathrm{n}=12$ )

(Fig 35) 6

5' - Tubérculos cefálicos grandes, em forma de duna, brânquias grossas (Fig. 36) (diâmetro, $\overline{\mathrm{x}}=36,8 \mu$, $\mathrm{n}=12$ ) S. daltanhani

6 - Filamentos branquias se ramificando no mesmo nivel, próximo da base (Figs. 29, 35), tubérculos cefálicos arredondados, a maioria com constricção na base (Fig. 21) ....... S. "A"

6'- Filamentos branquias não se ramificando no mesmo nível (Fig. 28), tubérculos cefálicos retangulares, dentiformes Fig. 22) S. "6-B1"

7 - Espécies com 8 filamentos branquiais .

7'- Espécies com mais de 8 filamentos branquiais 10

8. - Casulo com projeção dorsal anterior (Fig. 33); filamentos branquiais longos (Fig. 30), 1,5 vezes ou mais o comprimento do corpo .......

S. goeldii

8'- Casulo sem projeção dorsal anterior (Fig. 34), filamentos branquiais menores do que 1,5 vezes o comprimento do corpo 9

9. - Todos filamentos branquiais se originando próximo à base das brânquias

S. perflavum

9' - Filamentos branquias não se originando na mesma altura (Fig. 31) ..... S. iracouboense

10- Espécie com mais de 150 filamentos branquiais (Figs. 37-38)

S. trombetense

10' - Espécies com 18 a 21 filamentos branquiais (Figs. 39-40) ...... 11

11 - Filamentos projetados anteriormente, sem formar uma roseta na base (Fig. 39) $S$. rorotaense

11'. - Filamentos originando-se próximos da base, formando uma roseta na base (Fig. 40)

S. maroniense

\section{Chave de identificação para larvas (larvas preservadas em Carnoy)}

1- Larvas sem papilas ventrais posteriores (Fig. 41) 2

1'- Larvas com papilas ventrais posteriores (Fig. 42) ......................... 7

2-Larvas grandes $(>6,0 \mathrm{~mm})$.... 3

2'- Larvas pequenas a medianas $(<5,5 \mathrm{~mm})$ 6

3 - Histoblasto branquial grande (> 1,5 mm) (Figs. 43-44) ................ 4

3'- Histoblasto branquial pequeno $(<1,5 \mathrm{~mm})$ (Fig. 45) 5

4- Larvas de coloração verde escura; histoblasto branquial in situ com numerosos filamentos finos, partindo de um tronco grosso (Fig. $43)$, dissecado com mais de 150 filamentos (Figs. 37-38)

S. trombetense

4' - Larvas de coloração verde clara; histoblasto branquial in situ com filamentos grossos visiveis (Fig. 44), dissecado com 8 filamentos ..... S. perflavum

5- Histoblasto branquial dissecado com 18-21filamentos não dispostos em ângulo aberto (Fig. 39) ....... S. rorotaense

5' - Histoblasto branquial dissecado com 18-21filamentos dispostos em ângulo aberto, ao redor de uma roseta basal (Fig. 40) ..... S. maroniense

6- Cápsula cefálica pigmentada 
dorsalmente, formando um anel de coloração escura (Fig. 46); gânglio subesofageano não pigmentado; sem setas visiveis no tegumento corporal; brânquias dissecadas com 8 filamentos (Fig. 31)

S. iracouboense

6'- Cápsula cefálica sem pigmentação definida dorsalmente; gânglio subesofageano pigmentado
(Fig. 47); com setas visíveis no tegumento corporal; brânquias dissecadas com 6 filamentos

S. argentiscutum

7 - Gânglio subesofageano pigmentado (Fig. 48); fenda gular em forma de V; brânquias dissecadas com 6 filamentos grossos $(>30 \mu)$

S. daltanhani

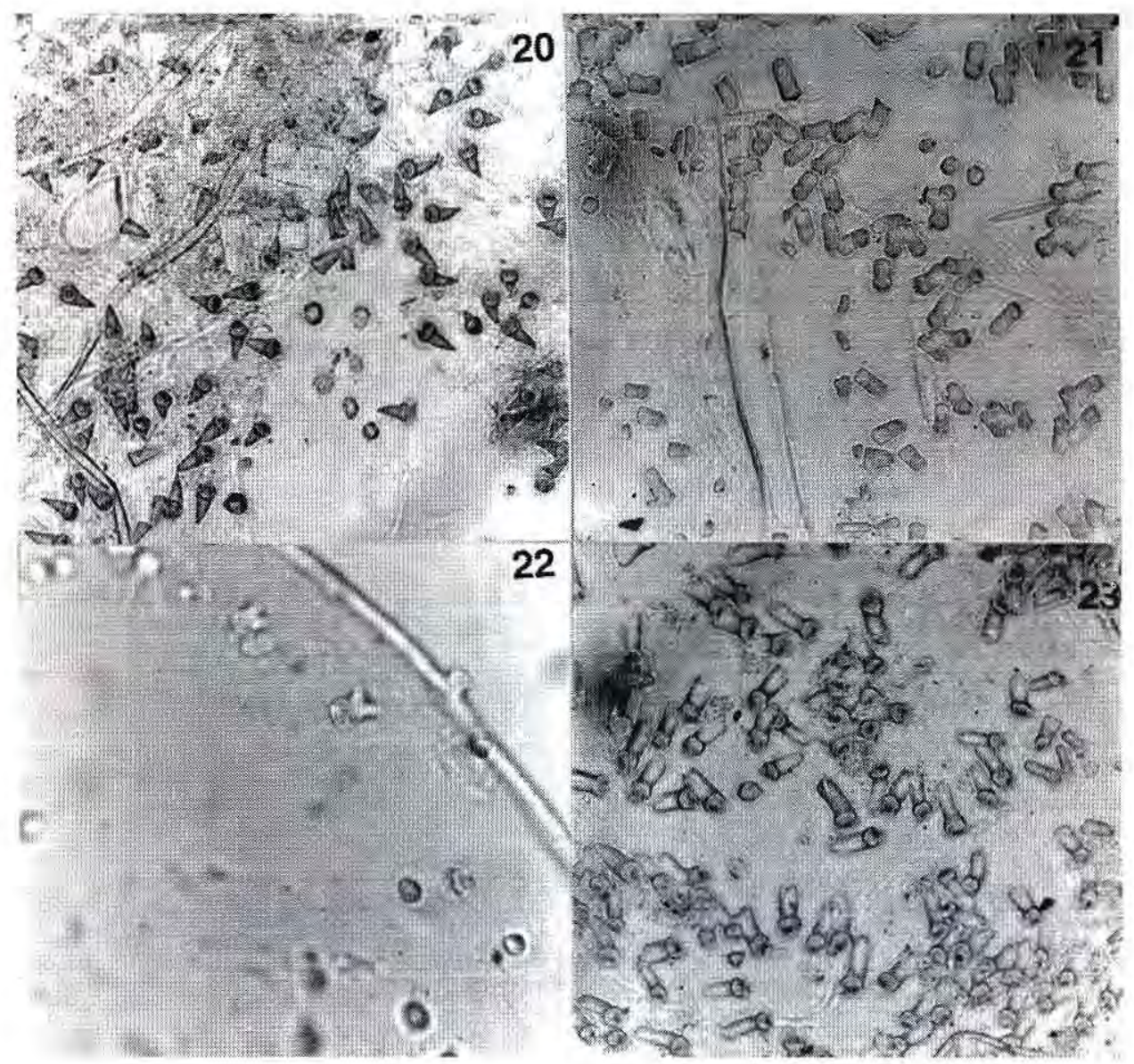

Figuras 20-23. Tubérculos cefálicos da pupa: Simulium quadrifidum (20); Simulium "A" (21); Simulium "6-B1" (22); Simulium goeldii (23). 

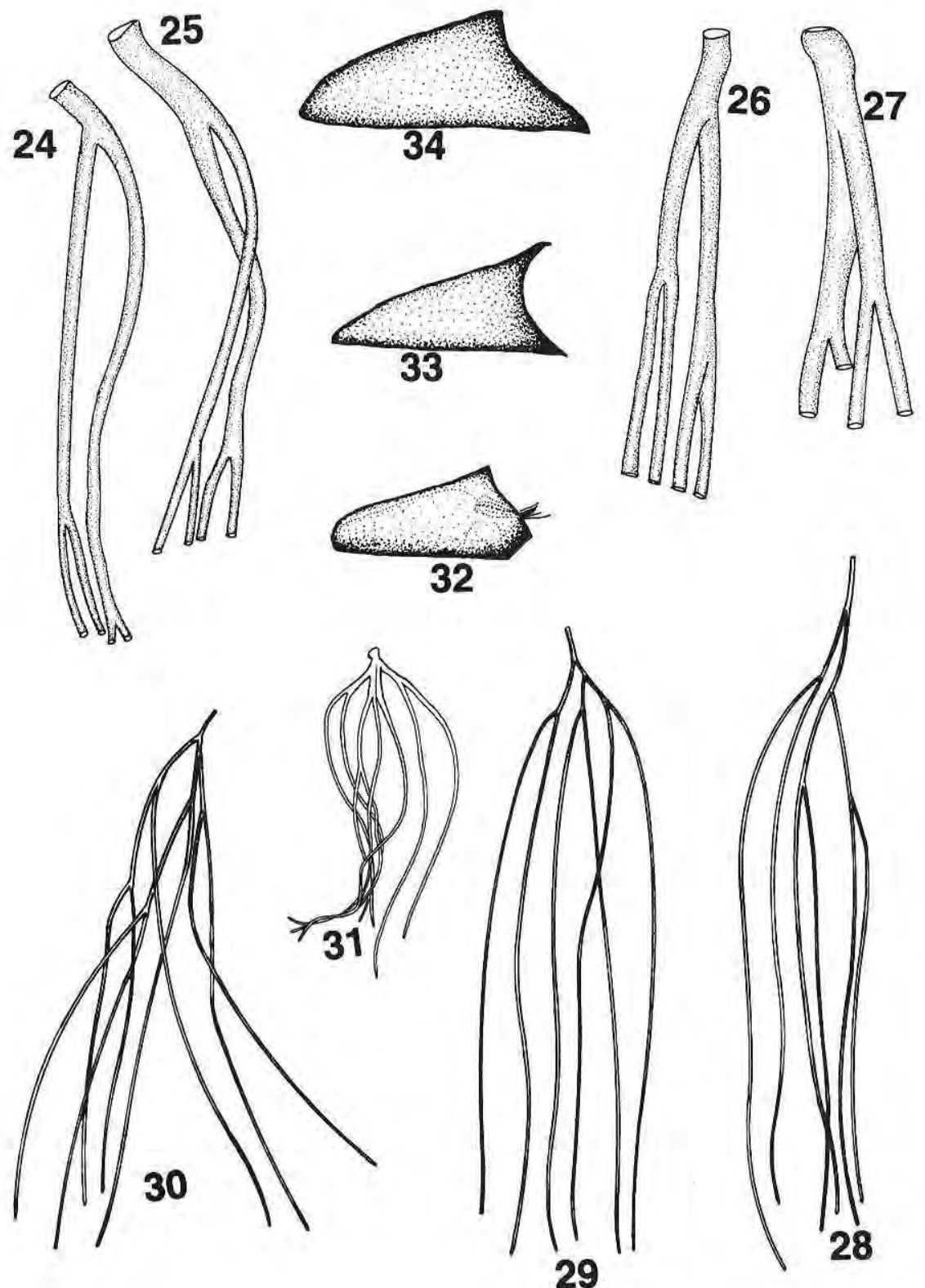

Figuras 24-34. Vista parcial dos filamentos branquias das pupas de Simulium cauchense (2425) e Simulium quadrifidum (26-27). Filamentos branquias das pupas de Simulium "6-B1" (28); Simulium "A" (29); Simulium goeldii (30); Simulium iracouboense (31). Casulo pupal em forma de bota (32). Casulo pupal em forma de chinelo com projeção anterior dorsal (33); sem projeção anterior dorsal (34). 


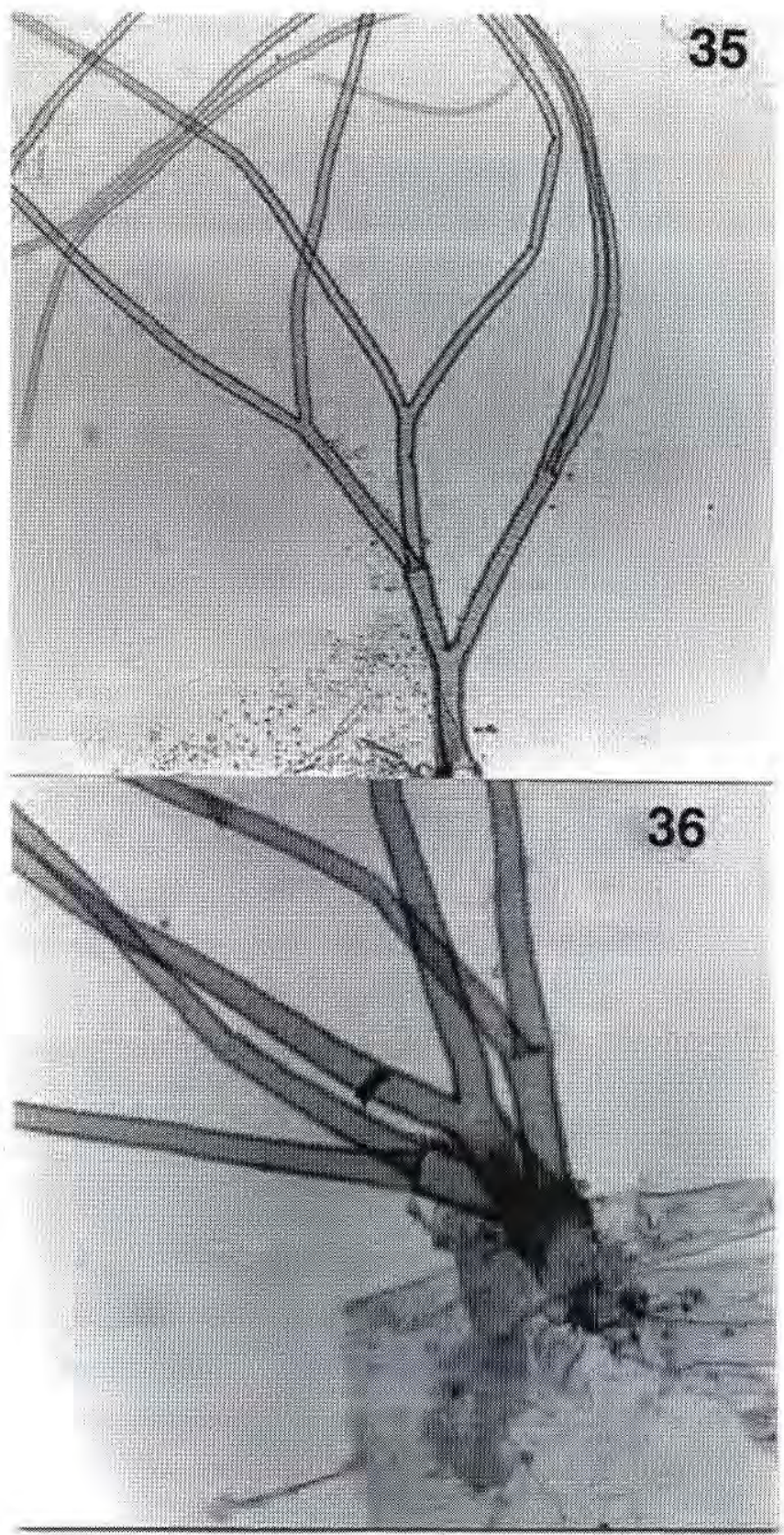

Figuras 35-36. Vista parcial dos filamentos branquiais das pupas de Simulium "A" (35) e Simulium daltanhani (36): 

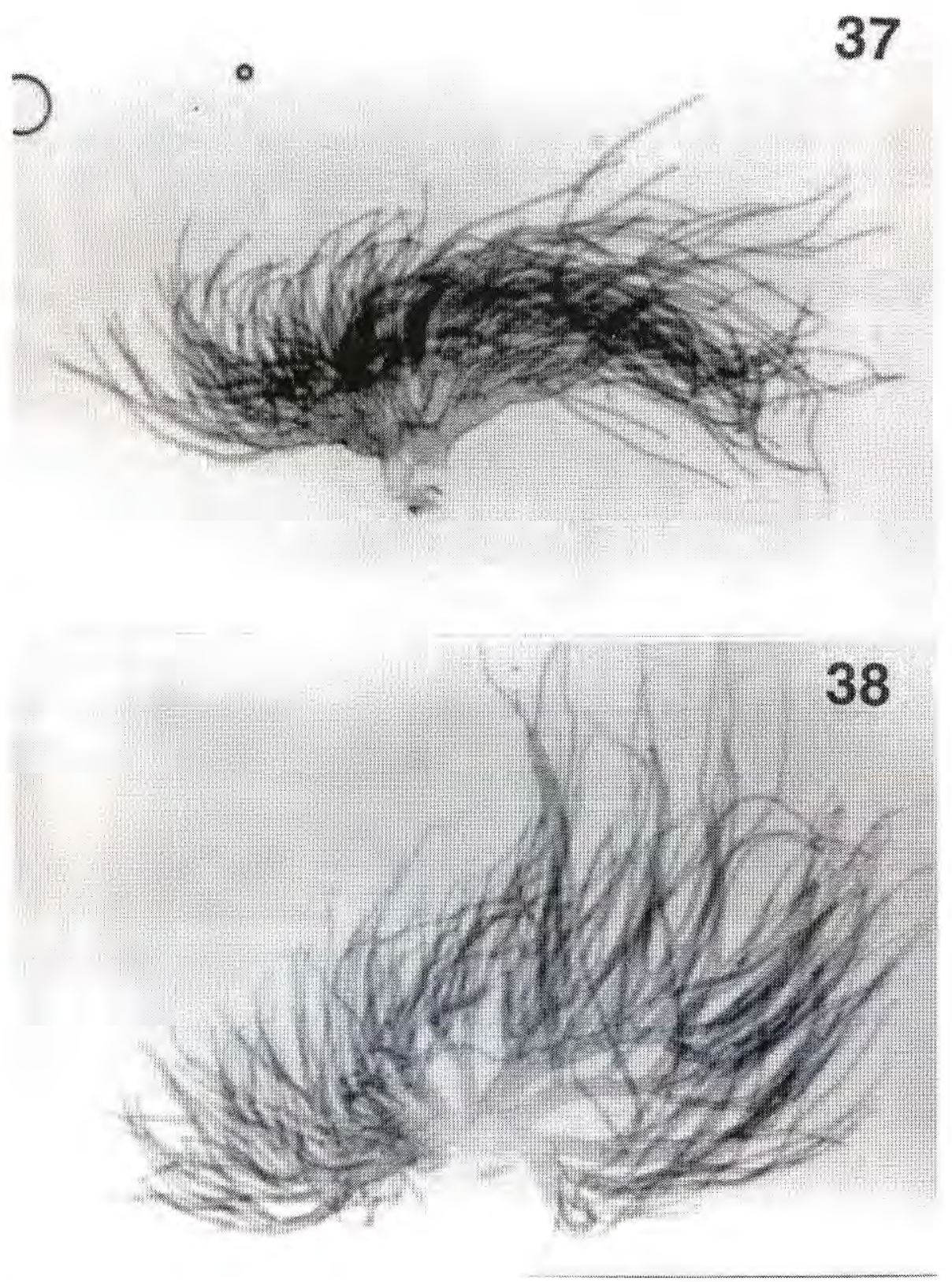

Figuras 37-38. Filamentos branquias da pupa de Simulium trombetense. 


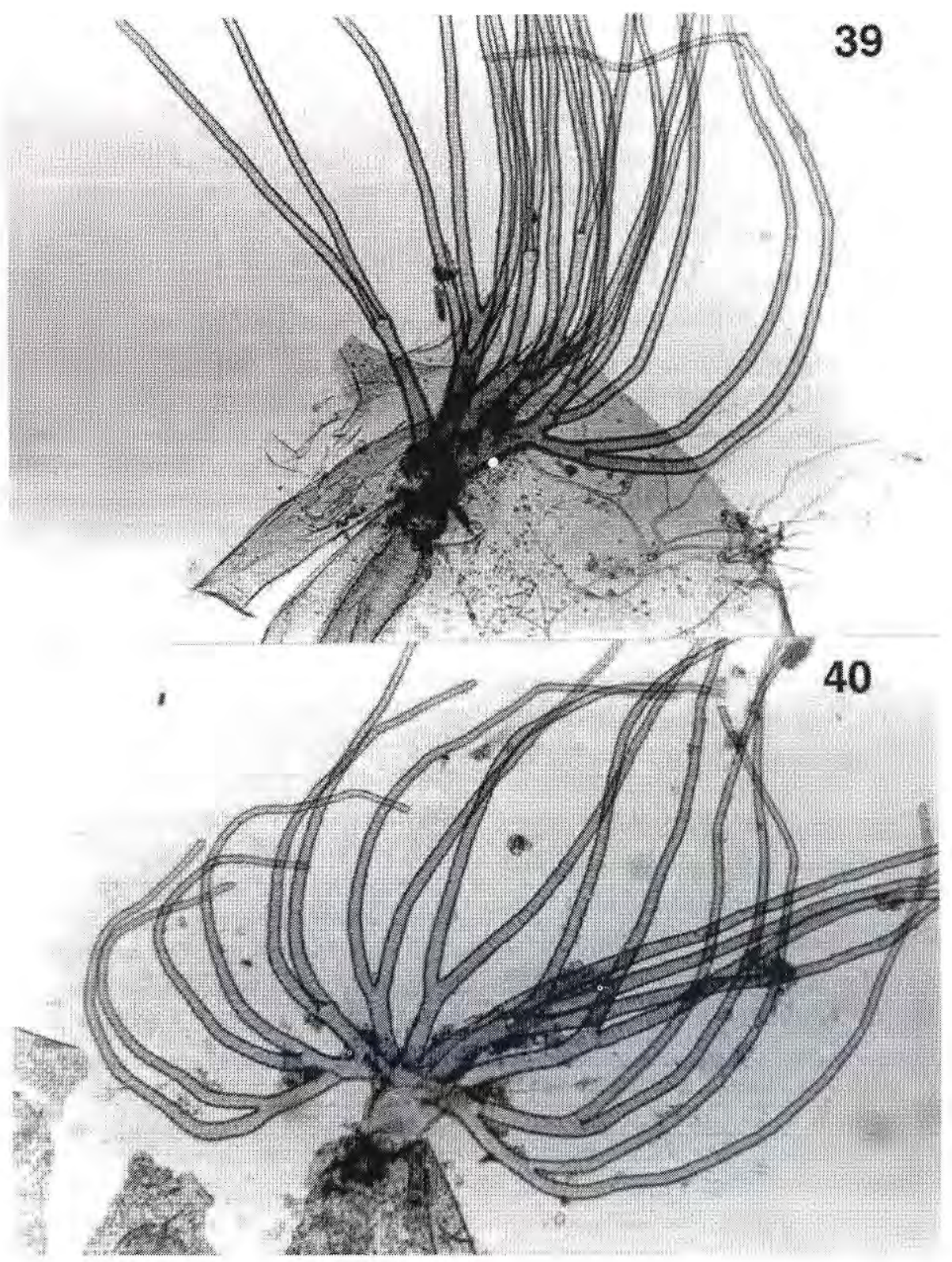

Figuras 39-40. Filamentos branquias da pupa de Simulium rorotaense (39) e Simulium maroniense (40). 
7'- Gânglio subesofageano não pigmentado; fenda gular arredondada; brânquias dissecadas com 4-8 filamentos delgados $(<25 \mu)$ 8

8 - Fenda gular com a porção apical alargada (Fig. 49); processo látero mandibular simples; segundo artículo antenal menor do que o primeiro ... 9

8 - Fenda gular com a porção apical não alargada (Fig. 50) processo látero mandibular bífido ou trífido; segundo artículo antenal maior do que o primeiro 10

9 - Larva com porção terminal de coloração azul escura, ou com bandas uniformes de coloração escura; setas pequenas, mas visiveis ao longo do corpo, raios do leque cefálico sem manchas escuras nos ápices do $1 / 4$ basilar, filamentos branquiais (dissecados) se ramificando longe da base (Figs. 24-25) S. cauchense

9' - Larva sem coloração azul escura na porção terminal, raios do leque cefálico, geralmente com manchas escuras nos ápices do $1 / 4$ basilar, filamentos branquiais (dissecados) se ramificando próximo da base (Figs. 26-27) S. quadrifidum

10 - Histoblasto dissecado com 8 filamentos branquiais (Fig. 30) ..... S. goeldii

$10^{\prime}$ - Histoblasto dissecado com 6 filamentos branquiais 11

11 - Histoblasto dissecado com filamentos se ramificando à mesma altura, próximo da base (Figs. 29; 35) ..... S. "A"

11'- Histoblasto dissecado com filamentos se ramificando a diferentes alturas (Fig. 28) S. "6-B1"

\section{DISCUSSÃO}

Diversos autores têm relatado a importância de fatores físico-químicos e da estrutura do habitat na distribuição das espécies (e.g. Grunewald, 1976; Corkum \& Currie, 1987; Ross \& Merritt, 1987; Ciboroswski \& Adler, 1990; Grillet \& Barreras, 1997; Hamada \& McCreadie, 1999). Os nossos resultados concordam com os desses autores, uma vez que podemos observar a formação de grupo de espécies de acordo com as caracteristicas físico-químicas do criadouro. Os imaturos de simulideos não podem selecionar o ambiente em que irão se desenvolver, dessa forma o habitat de cada espécie é selecionado pelas fêmeas, durante o processo de oviposição. A modificação do ambiente pela ação humana, como o desmatamento e a criação de represamentos d'água artificiais leva a dois resultados contrastantes. Do ponto de vista macro, essa modificação eleva a riqueza de espécies de uma região, permitindo que espécies consideradas invasoras, como $S$. perflavum e $S$. daltanhani (que não são encontradas em ambientes naturais na área de estudo) se estabeleçam nesses cursos d'água. Do ponto de vista micro, a modifícação do ambiente diminui a riqueza de espécies dos cursos d'água, uma vez que as espécies que colonizam os habitats naturais da região não colonizam esses cursos d'água, provavelmente em decorrência das alterações fisico-quimicas que não permitem as fêmeas reconhecerem o 
habitat alterado, como o ambiente para a oviposição.

As espécies $S$. maroniense e $S$. iracouboense foram coletadas apenas no igarapé Canoas, no município de Presidente Figueiredo, provavelmente localizado em uma área de transição entre o vale do Amazonas e o escudo guianense, uma vez que essas duas spécies, assim como S. cauchense, são mais abundantes no escudo guianense (Py-Daniel, 1983; Coscarón et al., 1996; Shelley et al., 1997; Hamada \& Adler, 1998b).

A distribuição de $S$. trombetense foi associada às bordas do escudo guianense (Hamada \& Adler, 1998b), e na Amazônia Central só foi encontrada, até o momento, nas áreas de transição entre o vale Amazônico e o referido escudo, no município de Presidente Figueiredo. As espécies $S$. goeldii e $S$. quadrifidum apresentam ampla distribuição na região Amazônica (Crosskey \& Howard, 1997). Simulium perflavum apresenta ampla distribuição na América do Sul (Coscarón, 1990), enquanto que $S$. daltanhani até o momento só foi registrada para a Amazônia Central (Hamada \& Adler, 1998a).

Simulium "6-B1" foi coletada por Dellome Filho (1978) em quatro igarapés no município de Manaus, e ainda não recebeu uma denominação formal, Py-Daniel (comunicação pessoal) afirma estar descrevendo essa espécie, uma vez que Dellome Filho (1978) depositou os espécimes estudados na coleção do INPA. Simulium " $A$ " representa, provavelmente, uma espécie nova para a Ciência, não registrada anteriormente na região, e será descrita pelos autores do presente estudo após estudos citológicos. Simulium goeldii, $S$. "6-B1" e $S$. "A" formam um grupo de espécies similares, que podem ser diferenciadas no estágio de pupa, pelo número e disposição dos filamentos branquias e no último estádio larval, após a dissecção do histoblasto branquial.

Simulium argentiscum (espécie antropofilica) tem ampla distribuição na região Amazônica (Crosskey \& Howard, 1997), entretanto, seus imaturos, até o momento, foram encontrados apenas no estado de Rondônia, nos rios Madeira (Shelley \& Luna Dias, 1980) e Mamoré (PyDaniel, 1983), cursos d'água com grande vazão. Mais amostragens nos grandes rios nas proximidades de Manaus serão necessárias para se entender a biologia e distribuição dessa espécie de importância médica, uma vez que ela é coletada picando seres humanos com certa freqüência, principalmente na época chuvosa, nos arredores de Manaus, como Vivenda Verde, Marchantaria, Puraquequara e no encontro das águas dos rios Negro e Solimões (J. Vidal e N. Hamada, observações pessoais).

Como a área ao sul do rio Amazonas foi pouco amostrada, é provável que mais espécies sejam adicionadas à lista apresentada aqui, quando as coletas de Simuliidae forem intensificadas nessa região.

\section{AGRADECIMENTOS}

Ao companheirismo e auxílio no campo de F.F. Xavier Filho, J. Bosco, 


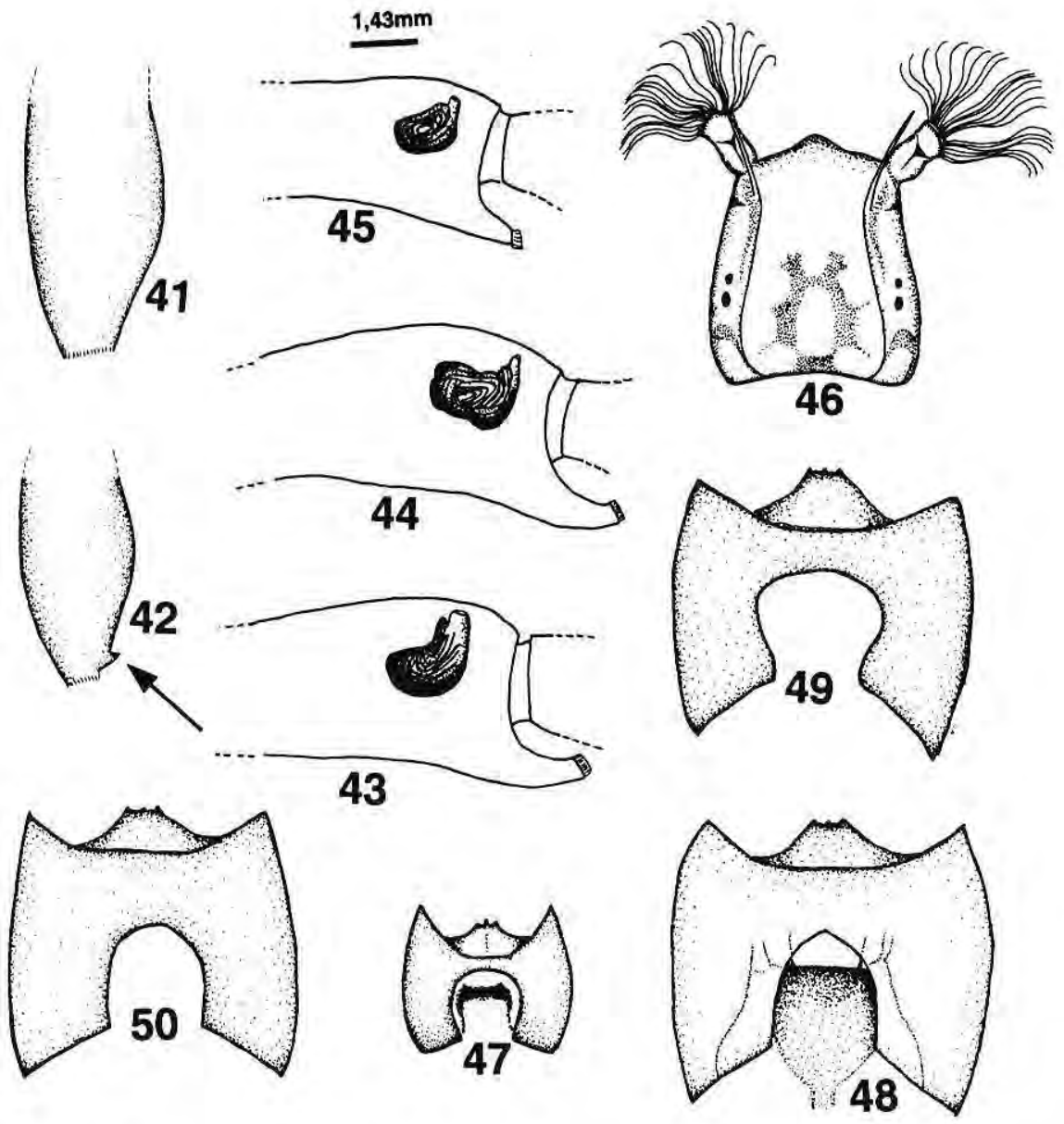

Figuras 41-50. Larvas. Porção terminal do corpo sem papilas ventrais posteriores (41) e com papilas ventrais posteriores (42). Histoblasto branquial in situ de Simulium trombetense (43); Simulium perflavum (44); Simulium rorotaense (45). Vista dorsal da cápsula cefálica de Simulium iracouboense (46). Fenda gular e gânglio subesofageano pigmentado de Simulium argentiscutum (47) e Simulium daltanhani (48). Fenda gular de Simulium quadrifidum (49) e Simulium goeldii (50).

L. Aquino, J.E. Bindá e U.C. Barbosa (INPA). À M. Maia-Herzog (FIOCRUZ) e A. Shelley (NHM-London), pelo empréstimo de parátipos de S. argentiscutum. P.M. Fearnside pela revisão do manuscrito. Deyse C.Q. Santos e Jorge Dácio ilustraram parte deste trabalho. Este trabalho foi parcialmente financiado pelo PPI 13400 , PPI 1-3070 (INPA), CNPq (201165/93-7). Especial agradecimento ao Comando Militar da Amazônia (CMA) e ao $6^{0}$ BIS (Rondônia) pelo total apoio de campo 
na área de Guajará-Mirim, que possibilitou a coleta de Simulium argentiscutum.

\section{Bibliografia citada}

Alencar, Y.B. 1998. Alimentação $e$ determinação biométrica de larvas de Simulium perflavum Roubaud, 1906 (Diptera: Simuliidae) em igarapés na Amazônia Central, Brasil. Dissertação de mestrado, INPA/UA, Manaus, AM, Brasil, $146 \mathrm{p}$.

Cerqueira, N.L.; Nunes de Mello, J.A. 1967. Simuliidae da Amazônia II. Descrição de Simulium goeldii sp. n. Amazoniana, 1: 125-130.

Cerqueira, N.L.; Nunes de Mello, J.A. 1968. Simuliidae da Amazônia IV. Descrição de Simulium fulvinotum sp. n. (Diptera, Nematocera). Amazoniana, 1(3): 205-210.

Ciborowski, J.J.H.; Adler, P.H. 1990. Ecological segregation of larval black flies (Diptera: Simuliidae) in northern Saskatchewan, Canada. Can. J. Zool., 68: 2113-2122.

Corkum, L.D.; Currie, D.C. 1987. Distributional patterns of immature Simuliidae (Diptera) in northwestern North America. Freshwater Biol., 17: 201-221.

Coscarón, S. 1990. Taxonomia y distribution del subgênero Simulium (Ectemnaspis) Enderlein (Simuliidae, Diptera, Insecta). Iheringia, Sér. Zool., 70:109-170.

Coscarón, S.; Ibañez-Bernal, S.; CoscarónArias, C.L. 1999. Revision of Simulium (Simulium) in the Neotropical realm (Insecta: Diptera: Simuliidae). Memoirs on Entomology International, 14: 543-604.

Craig, D.A. 1987. Some of what you should know about water or K.I.S.S. for hydrodynamics, Bull. N.A. Benthol. Soc., 35:178-182.

Crosskey, R.W.; Howard, T. M., 1997. A New Taxonomic and Geografical Inventory of World Blackflies (Diptera:Simuliidae), Department of Entomology, The Natural History Museum, London. 144p.

Dellome Filho, J. 1978. Fatores fisicos- quimicos dos criadouros de Simuliidae (Diptera, Nematocera). Dissertaçào de mestrado, INPA/FUA, Manaus, AM, Brasil, $75 \mathrm{p}$.

Dellome Filho, J. 1983. Consideraçôes sobre os fatores físico-químicos dos criadouros de Simulium goeldii Cerqueira \& Mello, 1967. (Diptera-Simuliidae). Revta Bras. Ent, 27:155-160.

Floch, H,; Abonnenc, E. 1946. Simulides de la Guyane Francaise. I. S. guianense Wise $1911, S$. rorotaense n. sp., $S$. maroniense n. sp. Publ. Inst. Pasteur Guyane Franc. et. Terr de L'Inini, 136: 1-20, 10pls.

Gorayeb, I.S.; Mok, W,Y. 1982. Comparison of capillary tube and immunodiffusion precipitin tests in the detection of Simulium fulvinotum larval predators, Ciência $e$ Cultura, 34: 1662-1668.

Gorayeb, I.S.; Pinger, R.R. 1978. Detecção de predadores naturais das larvas de Simulium fulvinotum Cerq. e Mello, 1968 (Diptera, Nematocera). Acta Amazonica, 8: 629-637.

Grillet, M.E.; Barreras, R. 1997. Spatial and temporal abundance, substrate partitioning and species co-occurrence in a guild of Neotropical blackflies (Diptera: Simuliidae). Hydrobiologia, 345(2-3): 197-208.

Grunewald, J. 1976. The hydro-chemical and physical conditions of the environment of the immature stages of some species of the Simulium (Edwardsellum) damnosum complex (Diptera). Tropenmed. Parasitol., 27: 438-454.

Hamada, N. 1989. Aspectos ecológicos de Simulium goeldii (Diptera: Simuliidae) Relação entre substrato e densidade de larvas. Mem. Inst. Oswaldo Cruz, 84: 263 266.

Hamada, N. 1993a. Association between Hemerodromia sp. (Diptera, Empididae) and Simulium perflavum (Diptera: Simuliidae) in Central Amazonia, Brazil. Mem. Inst, Oswaldo Cruz, 88: 169-170.

Hamada, N. 1993b. Abundância de larvas de Simulium goeldii (Diptera: Simuliidae) e 
caracterizaçào do seu habitat, em uma floresta de terra firme, na Amazônia Central. Bol. Mus. Para. Emilio Goeldi, sér. Zool., 9(2): 203-218.

Hamada, N. 1998. Bionomics of Simulium perflavum Roubaud (Diptera: Simuliidae) in Central Amazonia, Brazil. Rev. Bras. Ent., 41(2-4):523-526.

Hamada, N.; Adler, P.H, 1998a. A new species of Simulium (Diptera: Simuliidae) from open areas in Central Amazonia, Brazil. Mem. Inst. Osw. Cruz., 93(3): 317-325.

Hamada, N.; Adler, P.H. 1998b. Taxonomy of the Simulium perflavum Species-Group (Diptera: Simuliidae), with description of a new species from Brazil. Insecta Mundi, 12; 207-226.

Hamada, N.; Adler, P.H. 1999. Cytotaxonomy of four species in the Simulium perflavum Species-Group (Diptera: Simuliidae) from Brazilian Amazonia. Syst. Entomol., 24(2): 273-288.

Hamada, N.; McCreadie, J.W. 1999. Environmental factors associated with the distribution of Simulium perflavum (Diptera: Simuliidae) among streams in Brazilian Amazonia. Hydrobiologia, 397: 71-78.

Lacey, L.A.; Lacey, J.M. 1983. Filter feeding of Simulium fulvinotum (Diptera:Simuliidae) in the Central Amazon Basin. Quaest. Entomol., 19: 41-51.

Miranda Esquivel, D.R.; Muñoz de Hoyos, P. 1995. Ectemnaspis o Psilopelmia? He ahi el dilema. Revta Colomb. Entomol., 21(3): 129-144.

Moreira, G.R.P.; Sato, G. 1996. Black fly oviposition on riparian vegetation of waterfalls in an Atlantic rain forest stream. $A n$. Soc. Entomol. Brasil, 25(3): 557-562.

Py-Daniel, V. 1983. Caracterização de dois novos subgêneros em Simuliidae (Diptera: Culicomorpha) Neotropical. Amazoniana, $8(2): 159-223$.
Py-Daniel, V.; Sampaio, R.T.M. 1994. Jalacingomyia gen.n. (Culicomorpha): a ressurreição de Gymnopaidinae; a eliminação do nivel tribal; apresentação de novos caracteres e a redescrição dos estágios larval e pupal de Simulium colombaschense (Fabricius, 1787) (Diptera: Simuliidae). Mem. CAICET, IV: 101-148.

Ross, D.H.; Merritt, R.W. 1987. Factors affecting larval black fly distributions and population dynamics. In: Kim, KC; Merritt, R.W. (eds.), Black flies: Ecology,population management and annotated world list. The Pennsylvania State University, University Park, Pennsylvania, USA. pp.90-108.

Rothfels, K.H.; Dunbar, T.W. 1953. The salivary gland chromosomes of the black fly Simulium vittatum Zett. Can, J. Zool., 31: 226-241.

Shelley, A.J.; Lowry, C.A.; Maia-Herzog, M.; Luna Dias, A.P.A.; Moraes, M.A.P. 1997. Biosystematic studies on the Simuliidae (Diptera) of the Amazonia onchocerciais focus, Bull. Nat. Hist. Mus. Lond. (Ent.), 66(1): 1-121.

Shelley, A.J.; Luna Dias, A.P.A.. 1980. Simulium argentiscutum sp. nov. (Diptera: Simuliidae), a member of the $S$. amazonicum-group of species: description of adults, pupa and larva. Mem. Inst. Oswaldo Cruz, 75: 105-111.

Zar, H, J. 1996. Biostatistical Analysis, Editora Prentice-Hall do Brasil, Ltda., Rio de Janeiro, 662p. 